\title{
Health Advocate
}

\section{Carlos Beat Quinto}

Dr. med., Mitglied des FMH-Zentralvorstandes, Departementsverantwortlicher Public Health und Gesundheitsberufe

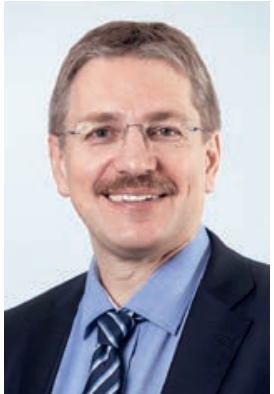

Im März 2017 beschlossen die medizinischen Fakultäten der Schweiz die Einführung von PROFILES (Principal Relevant Objectives and a Framework for Integrative Learning and Education in Switzerland). Eine der zentralen sieben Rollen, die Ärztinnen und Ärzte demnach einzunehmen haben, ist die des «Health Advocate». Sie sollen "Anwälte der Gesundheit» sein. Dies betrifft sowohl auf der individuellen Ebene das gesundheitsfördernde Verhalten als auch auf öffentlicher Ebene die gesundheitsfördernden Verhältnisse und Bedingungen. Dass bezüglich Verhältnisse in der Schweiz noch grosser Handlungsbedarf besteht, ist Thema der diesjährigen Swiss Public Health Conference «Kinder- und Jugendgesundheit» in Winterthur. Medizin darf sich nicht auf eine politische Ideologie verkürzen lassen. Das Manifest für Kinder- und Jugendgesundheit wird von der FMH und mehreren medizinischen Fachgesellschaften mitgetragen. Die Schweiz hat die Kinderrechtskonvention 1997 ratifiziert. Während Organisationen wie MSF im Sinne dieser Konvention handeln, verstossen gleichzeitig in der Schweiz domizilierte ausländische Konzerne gegen ebendiese.

\section{Hoffentlich bleibt das Tabakproduktegesetz eine Gesundheitsdebatte und entwickelt sich nicht zu einer Debatte um Werbeverbote.}

Entsprechend fand denn auch Mark Pieth, Professor für Strafrecht an der Universität Basel und international anerkannter Spezialist für Korruption, in der Synapse 1/2019 (www.synapse-online.ch) deutliche Worte betreffend Tabakproduktegesetz. Gemäss Professor Pieth gibt es eine einfache Lösung: Transparenz. Politiker und Politikerinnen sollten Offenlegungspflichten bezüglich Einkünfte und geldwerter Vorteile unterstehen, die im Zusammenhang mit ihrem politischen Mandat stehen. Tausende Kinder und Jugendliche im In- und Ausland werden durch von der Schweiz aus operierende internationale Konzerne in die Nikotinabhängigkeit geführt. Die gesetzlichen Vorgaben der Schweiz betreffend Tabakwerbung und -sponsoring und Abgabe von Tabak und nikotinhaltigen Produkten an Kinder und Jugendliche sind die rückständigsten auf dem europäischen Kontinent. Umso mehr begrüsst die FMH den Entscheid der Gesundheitskom- mission des Ständerats, eine Verschärfung der Bestimmungen betreffend Tabakwerbung herbeizuführen. Wenn auch die FMH gerne eine stärkere Verschärfung sehen würde, um den Anschluss an die über 180 Staaten nicht zu verpassen, welche die WHO-Rahmenkon-

Tausende Kinder und Jugendliche werden durch von der Schweiz aus operierende Konzerne in die Nikotinabhängigkeit geführt.

vention bereits ratifiziert haben, so erachtet die FMH den Entscheid der SGK-S doch als wichtigen Schritt in die richtige Richtung. Die Regulierung des Zugangs zu Tabakprodukten bleibt eines der präventivmedizinisch wichtigsten Themen.

Es bleibt zu hoffen, dass die Ausgestaltung des Tabakproduktegesetzes eine Gesundheitsdebatte bleibt und sich nicht zu einer ordnungspolitischen Debatte um Werbeverbote und Werberecht entwickelt. Denn die Hälfte der tabakkonsumierenden Kinder und Jugendlichen wird voraussichtlich an den Folgen ihres Konsums sterben, egal ob Zigarette oder Heated Tobacco Product (HTP) [1] - allerdings nicht ohne der ausländischen Tabakindustrie zuvor Milliardengewinne ermöglicht zu haben und zugleich Milliardenkosten für die Gesundheitswesen zahlreicher Länder inklusive der Schweiz zu verursachen. Der Ärzteschaft werden dann diese politisch verantworteten Kosten vorgehalten. Das in Deutschland bereits als untaugliches Mittel erkannte Globalbudget, welches von der deutschen Regierung in Raten bereits wieder abgeschafft wird, soll in der Schweiz unter der Bezeichnung "Zielvorgaben" oder "degressive Tarife» eingeführt werden. Es stellt, wie man in Nachbarländern sieht, den Königsweg in eine Zweiklassenmedizin dar, führt $\mathrm{zu}$ einer massiven Zunahme des administrativen Aufwandes und ist daher auch aus Public-Health-Sicht abzulehnen.

Mit PROFILES werden wir zukünftig mehr Kolleginnen und Kollegen ausbilden müssen, die sich als «Health Advocates» engagieren. Denn es braucht mehr Transparenz und medizinischen Sachverstand, insbesondere gegen «Fake News», in der Schweizer Gesundheitspolitik. Unterstützen wir in diesem Sinne unsere Kolleginnen und Kollegen, die im Herbst für die nationalen Parlamentswahlen kandidieren. 